\title{
Ultrabroadband Electro-Optic Modulator Based on Hybrid Silicon-Polymer Dual Vertical Slot Waveguide
}

\author{
Shouyuan Shi and Dennis W. Prather \\ Department of Electrical and Computer Engineering, University of Delaware, 140 Evans Hall, Newark, DE 19716, USA \\ Correspondence should be addressed to Shouyuan Shi, sshi@ee.udel.edu
}

Received 15 May 2010; Accepted 30 July 2010

Academic Editor: Ana Vukovic

Copyright (C) 2011 S. Shi and D. W. Prather. This is an open access article distributed under the Creative Commons Attribution License, which permits unrestricted use, distribution, and reproduction in any medium, provided the original work is properly cited.

\begin{abstract}
We present a novel hybrid silicon-polymer dual slot waveguide for high speed and ultra-low driving voltage electro-optic (EO) modulation. The proposed design utilizes the unique properties of ferroelectric materials such as $\mathrm{LiNbO}_{3}$ to achieve dual $\mathrm{RF}$ and optical modes within a low index nanoslot. The tight mode concentration and overlap in the slot allow the infiltrated organic EO polymers to experience enhanced nonlinear interaction with the applied electric field. Half-wavelength voltage-length product and electro-optic response are rigorously simulated to characterize the proposed design, which reveals ultrabroadband operation, up to $250 \mathrm{GHz}$, and subvolt driving voltage for a $1 \mathrm{~cm}$ long modulator.
\end{abstract}

\section{Introduction}

Low driving voltage and high-speed electro-optic (EO) modulators are of great interest due to their wide variety of applications including broadband communication, RF photonic links, millimeter wave imaging, and phased-array radars. In order to attain optical modulation at low driving voltages, a strong mode concentration and a tight mode overlap between optical and radio-frequency (RF) modes in the nonlinear EO material are required. Typically, to maintain a single mode operation in optical domain, the optical mode size is on an order of wavelength, that is, $2 \mathrm{um}$ at telecommunication region. As a result, to match with the optical mode, the RF guiding structure essentially has to reduce a factor of three orders of magnitude. Conventional traveling wave EO modulators are usually driven by RF transmission lines, such as coplanar waveguides (CPWs) and microstrip lines. These electrode designs provide not only high speed operation but also a strong overlap between optical and RF modes. While the device operates at very high frequency, that is, over $20 \mathrm{GHz}$, the RF wave propagation attenuation attributed from both conduction loss and dielectric loss becomes the key issue that prevents the device from operating over a wide bandwidth. Physically, a small mode size provides a strong RF field concentration, or a small mode volume, however, leads to a significant increase in propagation loss. As a result, an optimal design of RF electrode design including signal electrode and gap between signal and ground is required to minimize the overall RF propagation loss.

To date, many high speed traveling wave EO modulators have been designed, fabricated, and characterized, leading to operation at speeds as high as $140 \mathrm{GHz}$. Most of these modulators were developed using crystalline EO materials, such as $\mathrm{LiNbO}_{3}[1-5]$ and $\mathrm{GaAs}[6,7]$. Recently, tremendous efforts have gone into the development and optimization of organic EO polymers. State-of-the-art nonlinear electrooptic polymers have been reported to have an EO coefficient of $r_{33}=100 \mathrm{pm} / \mathrm{V}$ or higher [8-14], which is nearly an order of magnitude higher than the crystalline $\mathrm{LiNbO}_{3}$ and two orders of magnitude higher than most III-V materials. With the recent breakthroughs in silicon photonics, specifically vertical and horizontal slot waveguides $[15,16]$, the silicon organic hybrid represents a tremendous opportunity to develop highly sensitive devices for an array of photonics applications [17-20]. A variety of designs including ridged slot waveguides and segmented slot waveguides have been proposed for modulation applications [21-24]. By placing low index EO polymer material in the slot region, the optical mode will be tightly confined in the nonlinear material. This requires an extremely small RF mode concentration 
for deep modulation. One of the approaches is to dope the silicon ridges at the edge of the slot; the voltage then will be applied directly across the slot region, thereby maximizing the electric field. This confinement of the optical mode and electric field allows for modulation at millivolt driving voltages $[22,23]$. However, the introduction of doping in the silicon ridges also induces significant RF propagation loss. The loss is strongly dependent on the operational frequency and the doping level but can reach values up to $30 \mathrm{~dB} / \mathrm{cm}$ at $100 \mathrm{GHz}$. To mitigate the RF loss, a novel modulator design based on a dual horizontal slot waveguide, where a unique ferroelectric material is used to form an $\mathrm{RF}$ and optical nanoslot in a microstrip line with a large electrode separation, has been proposed. An ultra-high speed modulation and extremely low driving voltage are achieved [25]. In this paper we present a CMOS compatible vertical dual slot waveguide EO modulator design, where the slot waveguide is formed on silicon-on-insulator wafer.

In order to accurately characterize the proposed device, both optical and RF analysis are performed by using vectorial waveguide mode solvers. In the optical domain, a Finitedifference Frequency-domain (FDFD) method is employed [26]. On the other hand, due to nanometer scale of slot structure, the analysis of such a device at RF frequency becomes extremely challenging. To this end, a finite-element method (FEM) based commercial software package, ANSYS High Frequency Surface Simulation (HFSS), was employed. The adaptive meshing scheme allows handling both very subwavelength and larger features to achieve convergent results. In the rest of paper we will present the slot waveguide analysis and design, as well as its electro-optic characterization in terms of sensitivity and high frequency response.

\section{Dual RF Photonic Slot Waveguide}

The dual vertical slot waveguide is formed on a silicon-oninsulator (SOI) substrate. As depicted in Figure 1, the device consists of two ridges with a high refractive index, that is, silicon, and a nanometer-scale slot infiltrated with a lower refractive index EO polymer material that is sandwiched between the ridges. The silicon ridges then are bounded with ferroelectric materials, such as $\mathrm{LiNbO}_{3}$ and $\mathrm{TiO}_{2}$. The nanoslot has a gap size of $w_{p}$ and silicon ridges have a width of $w_{s i}$. The outer claddings have a width of $w_{c}$ and their height is aligned with silicon substrate. The polymer is infiltrated in the slots as well as the gaps between signal electrodes and ground of coplanar waveguide. As shown in Figure 1, by introducing an RF coplanar waveguide, two sets of slot waveguides can be embedded in the gaps, thereby forming a push-pull EO modulator configuration. The gold electrodes have a signal width of $w_{m}$ and thickness of $h_{m}$. In this design a SOI wafer with thickness of $h_{s i}=260 \mathrm{~nm}$ and an oxide layer of $2 \mu \mathrm{m}$ are used, thereby leading to a single mode operation in the vertical direction at telecommunication wavelengths.

The ferroelectric materials possess unique material properties, that is, low optical refractive indices and very high RF dielectric constants. In particular, $\mathrm{LiNbO}_{3}$ material is used to form the waveguide due to its additional EO effect

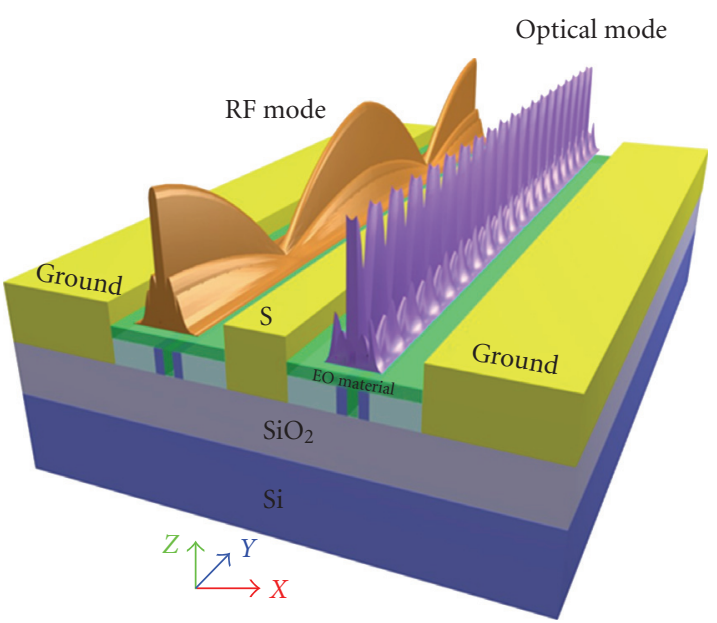

(a)

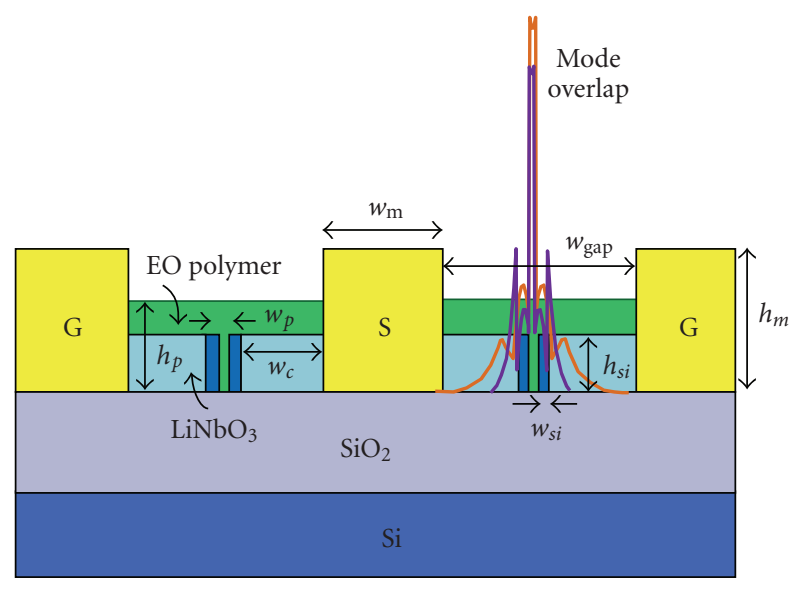

(b)

FIGURE 1: Dual vertical slot waveguide EO modulator. (a) Schematic layout of dual nanoslot waveguides embedded in the CPW, where three-dimensional RF and optical mode profiles in the middle plane along the waveguide propagation direction are shown on the top of slot waveguide. (b) Cross-sectional view of dual slot waveguide, where strong mode confinement and overlap are illustrated in the center slot.

on the modulation. In the optical wavelength range, that is, at an operation wavelength of $1.55 \mu \mathrm{m}$, the ordinary and extraordinary refractive indices of $\mathrm{LiNbO}_{3}$ are $n_{0}=2.214$ and $n_{e}=2.138$, respectively. However, in the RF frequency range, the anisotropic $\mathrm{LiNbO}_{3}$ substrates have high relative permittivities of 28 and 43 perpendicular and parallel to the substrate surface. Silicon ridges have an RF relative dielectric constant of 11.97, which is a slight variation compared to their optical refractive index of 3.5. The EO polymer has an optical refractive index of 1.6 and an RF relative dielectric constant of 2.49. Obviously, the indices of $\mathrm{LiNbO}_{3}$ are lower than that of silicon at optical wavelengths and much greater than that of silicon in the RF frequency range. These unique material properties allow us to construct a dual nanoslot waveguide in order to achieve both optical and RF mode concentration. 


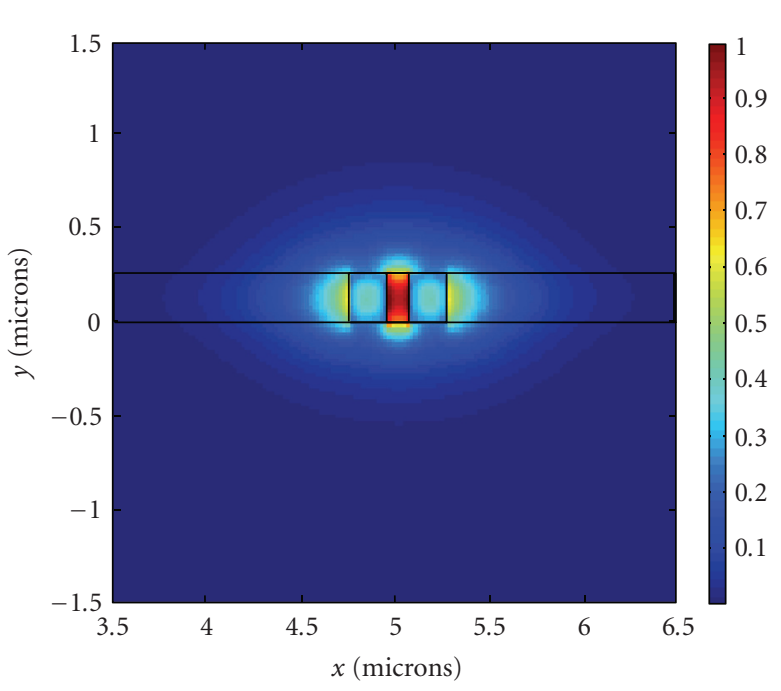

(a)

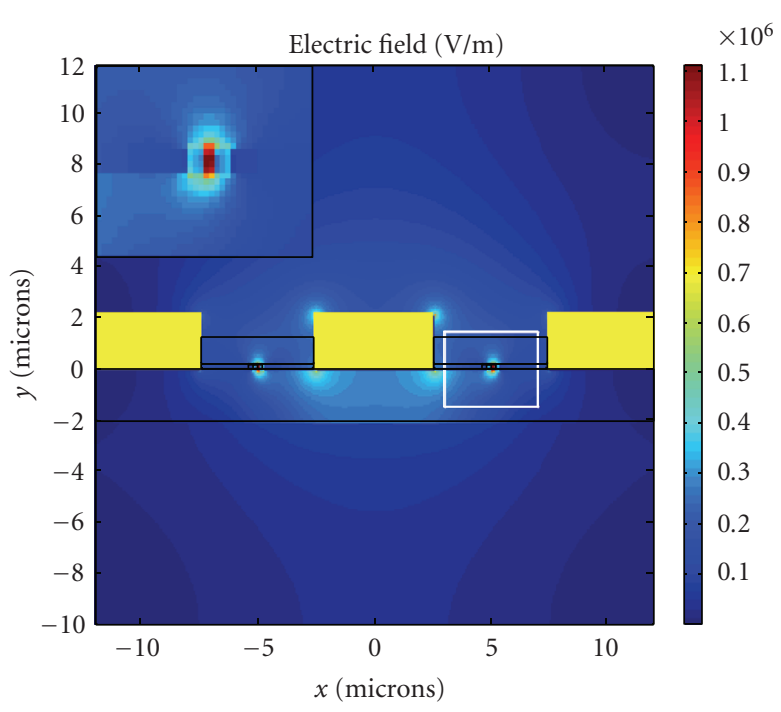

(b)

Figure 2: Optical and RF mode profiles. (a) Optical electric field distribution at a wavelength of $1.55 \mu \mathrm{m}$. (b) RF electric field distribution at a frequency of $100 \mathrm{GHz}$ with $1 \mathrm{~V}$ applied between the signal electrode and ground. The colorbar scale confirms the field concentration near $1.1 \mathrm{MV} / \mathrm{m}$ within the low index EO polymer infiltrated slot. The dashed box indicates the position of the optical mode as shown in (a).

First, consider a quasitransverse electric (TE) mode in the optical domain, where the dominant electric field component is parallel to the horizontal plane, as shown in Figure 1. The electromagnetic boundary conditions reveal the electric field discontinuity across the slot, thereby resulting in an enhancement in the electric field strength in the low index material [15]. In order to characterize optical confinement within the polymer material, the confinement factor is defined as the ratio of the propagation power inside the slot to the total power of the guiding mode, $\Gamma_{o}=\int_{\text {Slot }} \operatorname{Re}(\mathbf{E} \times \mathbf{H}) d s / \int_{\text {Waveguide }} \operatorname{Re}(\mathbf{E} \times \mathbf{H}) d s$. The figure of merit, $\Gamma_{o}$, is strongly dependent on the design parameters, such as waveguide height, slot width, and silicon ridge width. Therefore, optimization of the design parameters is required to maximize mode confinement factor to achieve an improved EO modulation.

A full-vectorial finite-difference (FD) analysis [26] that is capable of handling anisotropic and lossy material properties is developed and employed to simulate the waveguiding characteristics, that is, the guided mode and effective index. We consider a slot waveguide with a gap size of $w_{p}=120 \mathrm{~nm}$ and a silicon ridge width of $w_{s i}=250 \mathrm{~nm}$. The EO polymer has a total height of $h_{p}=1.2 \mu \mathrm{m}$. Figure 2(a) shows the optical electric field distribution at the wavelength of $1.55 \mu \mathrm{m}$ with an effective index of 2.027. Across the middle plane of the waveguide structure an enhancement of $\left(n_{s i} / n_{p}\right)^{2}=4.7$ in the electric field can be observed. Based on the mode profile, the calculated optical confinement factor in the EO polymer is $\Gamma_{o}=36.56 \%$. The large mode concentration in the nanoslot provides an opportunity for the enhanced nonlinear interaction with applied electric field. As shown in Figure 2(a), the further extension of optical mode into top cladding of $\mathrm{EO}$ polymer and edge cladding of $\mathrm{LiNbO}_{3}$ will lead to an improved EO modulation.
The strong optical mode confinement in the slot suggests a RF mode overlap in the slot to maximize the modulation. Due to the very extremely different wavelength scales between RF and optical waves, the design for RF mode in a nanometer-scale slot becomes extremely challenging. Efforts have been made to establish the electrical field in the vertical slot by highly doping the silicon. However, at high frequencies the doped silicon becomes extremely lossy rather than conductive, thereby prohibiting its high speed operation. With the application of $\mathrm{LiNbO}_{3}$ as the outer claddings, let's also concern with concentrating the RF signal. While an RF signal is applied to the CPW electrodes, a CPW mode is established in which the dominant electric field in the gap between signal and ground is aligned in the $x$ direction. Since $\mathrm{LiNbO}_{3}$ material has a much larger dielectric constant than the silicon and EO polymer materials, the boundary conditions reveal that the resultant RF electric field inside the ferroelectric and silicon materials is much weaker than within the EO polymer. The weak electric field in the high dielectric constant materials indicates a small fraction of voltage drop across the materials; as a result, a large portion of the applied voltage is directly across the slot. The resulting structure creates an effective RF nanoslot waveguide within the transmission line. Due to the strong mode confinement in the low index EO polymer infiltrated slot, a lower overall $\mathrm{RF}$ effective index can be achieved. In the proposed design, the signal electrode width is $w_{m}=8 \mu \mathrm{m}$ and the CPW gap size is $w_{\text {gap }}=6 \mu \mathrm{m}$. The electrode thickness, $h_{m}$, is optimized to match the RF effective index with that of the optical mode, enabling high speed operation. The loss tangents of $\mathrm{LiNbO}_{3}$ and silicon are 0.004 and 0.002 , respectively, and the conductivity of gold is $4.1 \times 10^{7} \mathrm{~S} / \mathrm{m}$. As shown in Figure 2(a), a strong electric field confinement, over $1.1 \times 10^{6} \mathrm{~V} / \mathrm{m}$, is observed when a voltage of $1 \mathrm{~V}$ is applied across the signal 


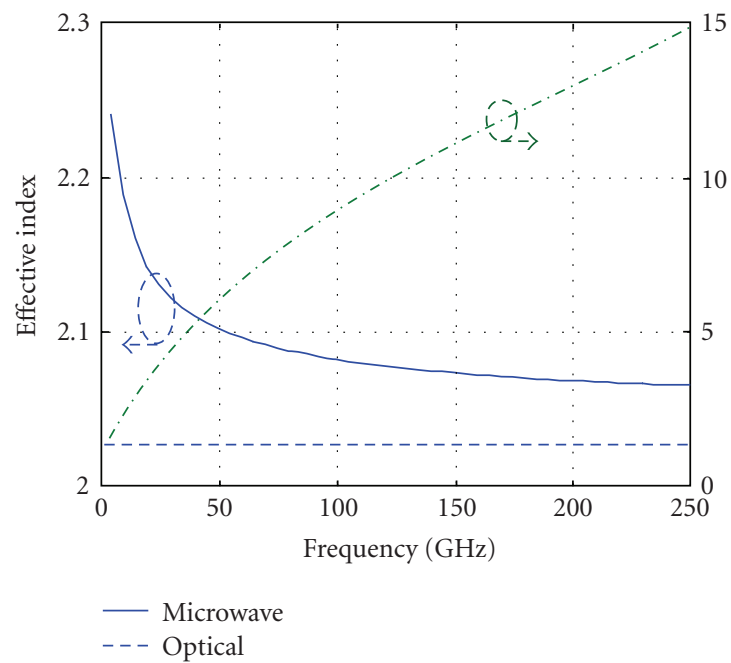

(a)

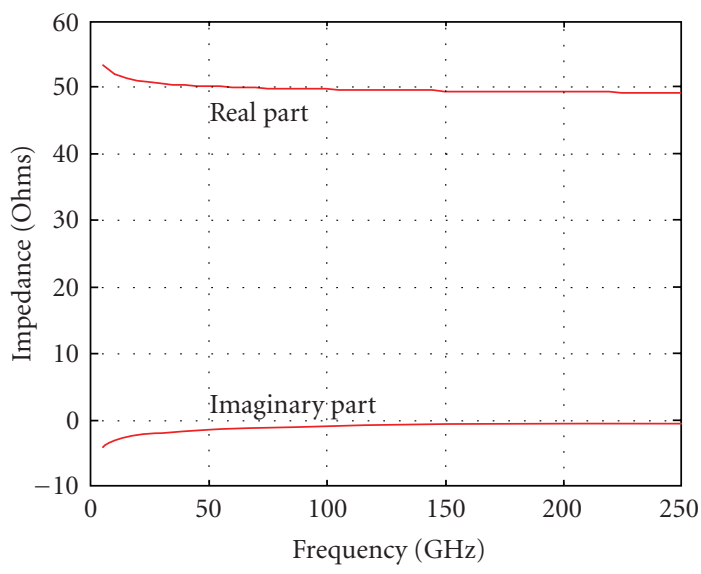

(b)

FIGURE 3: RF propagation characteristics of dual slot waveguide. (a) RF and optical effective indices and RF attenuation constant over the frequencies of $250 \mathrm{GHz}$. The dashed blue line indicates the effective optical index of 2.502. (b) Characteristic impedance.

electrode and ground at a frequency of $100 \mathrm{GHz}$. Such a strong electric field concentration will induce a significant optical index change in the nonlinear EO polymer material, resulting in a deep optical modulation. An ultrabroadband response that spans from $\mathrm{DC}$ to $250 \mathrm{GHz}$ is performed by HFSS.

On the other hand, the introduction of the high dielectric constant material potentially allows for an increased electrode separation between signal and ground without significantly decreasing the electric field confinement in the slot. As a result, the RF mode will experience reduced conduction loss. This becomes of particular importance for the modulators to operate at high frequencies. Figure 3(a) shows the comparison between the optical effective index and frequency dependent RF effective index, as well as the attenuation constant for the RF slot waveguide. An optimal electrode thickness, $h_{m}=2.2 \mu \mathrm{m}$, is found to minimize the index mismatch to a value as low as 0.038 at high frequencies. Due to tight concentration in the slot, the overall RF effective does not vary significantly, and tends to be convergent to 2.065 at the frequencies over $50 \mathrm{GHz}$. Figure 3(b) shows the real and imaginary parts of the characteristic impedance. The finite conductivity has a strong impact on the characteristic impedance. At frequencies over $50 \mathrm{GHz}$, the characteristic impedance remains to be $49 \mathrm{Ohms}$.

\section{Electro-Optic Modulation Analysis}

In order to characterize the high speed electro-optic response and sensitivity of the proposed device based on the simulated optical and RF guided mode characteristics in the previous section, consider a traveling wave electro-optic modulator, where both optical and RF guided modes are copropagated along the same direction over a length of $L$. The RF voltage amplitude along the transmission line can be expressed as $[4,27]$

$$
V\left(f_{m}, y\right)=V_{0} e^{-\alpha_{m}\left(f_{m}\right) y} e^{j\left(k_{m} \delta y-2 \pi f_{m} t_{0}\right)}
$$

where $V_{0}$ is the amplitude of the RF wave at the input port, $k_{m}=2 \pi n_{m} / \lambda_{m}$ is the RF wave number, $\alpha_{m}$ is the $\mathrm{RF}$ attenuation constant, $\delta=1-n_{o} / n_{m}$ is the relative index mismatch between RF effective index $n_{m}$ and optical effective index $n_{o}$, and $f_{m}$ is the RF frequency. Driven by the microwave, the optical phase induced at the end of modulator is derived by integrating the induced phase over the modulator, given by

$$
\Delta \phi\left(f_{m}\right)=\int_{0}^{L} \frac{2 \pi}{\lambda_{o}} \Gamma V_{0} e^{-\alpha_{m}\left(f_{m}\right) y} e^{j\left(k_{m} \delta y-2 \pi f_{m} t_{0}\right)} d y,
$$

where the mode overlap integral factor, $\Gamma$, that characterizes the induced changes in refractive index for a given applied voltage of $V_{0}$, is defined by the mode overlap integral between optical and RF electric fields in the cross section transversal to the modulator propagation direction,

$$
\Gamma=\frac{1}{V_{0}} \frac{\iint_{S}(1 / 2) n_{\mathrm{eff}}^{3} r_{33}(x, z) E_{m}(x, z)\left|E_{o}(x, z)\right|^{2} d s}{\iint_{S}\left|E_{o}(x, z)\right|^{2} d s},
$$

where $n_{\text {eff }}$ is the effective index of the optical mode, $r_{33}$ is the EO coefficient distribution, and $E_{m}$ and $E_{o}$ are the RF and optical electric fields, respectively. To characterize the highspeed response of the modulator, the electro-optic response of the modulator, derived from the induced phase at a given 


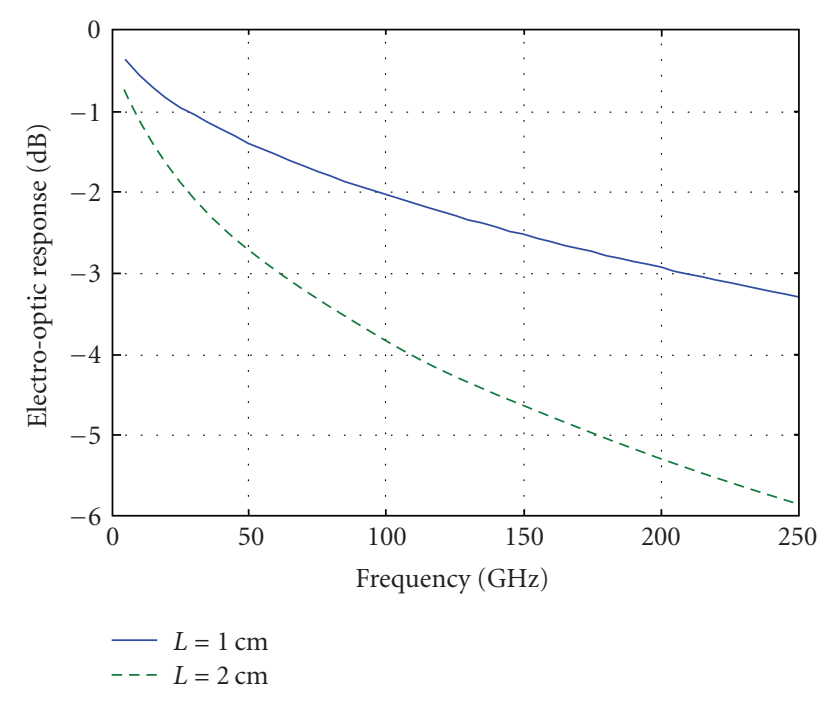

FIGURE 4: Electro-optical response of the proposed modulators with different modulator lengths of 1 and $2 \mathrm{~cm}$.

TABLE 1: DC half-wavelength length-product.

\begin{tabular}{lc}
\hline$r_{33}(\mathrm{pm} / \mathrm{V})$ & $\mathrm{DC} V_{\pi} L(\mathrm{mV} \cdot \mathrm{cm})$ \\
\hline 30 & 675.8 \\
100 & 215.5 \\
170 & 128.2 \\
\hline
\end{tabular}

RF frequency of $f_{m}$, is defined as a normalized quantity compared to the phase change at DC,

$$
\begin{aligned}
m\left(f_{m}\right) & =\frac{\left|\Delta \phi\left(f_{m}\right)\right|}{|\Delta \phi(0)|} \\
& =e^{-\alpha_{m}\left(f_{m}\right) L / 2} \sqrt{\frac{\sinh ^{2}\left(\alpha_{m} L / 2\right)+\sin ^{2}\left(k_{m} \delta L / 2\right)}{\left(\alpha_{m} L / 2\right)^{2}+\left(k_{m} \delta L / 2\right)^{2}}} .
\end{aligned}
$$

From the above equation, the electro-optic response is strongly dependent on the RF loss coefficient and index matching, or velocity matching, between optical and RF guided modes. The half-wavelength voltage of $V_{\pi}\left(f_{m}\right)$ corresponding to a phase change of $\pi$ over the interaction length of $L$ to produce a zero optical output can be derived as,

$$
V_{\pi}\left(f_{m}\right)=\frac{V_{\pi}(\mathrm{DC})}{m\left(f_{m}\right)},
$$

where DC half-wavelength voltage is given by the $V_{\pi}(\mathrm{DC})=$ $\lambda_{o} / 2 \Gamma L$. The longer the length of the modulator is, the lower the DC $V_{\pi}$ is; however, the lower the EO response is due to RF loss. Therefore, there is an optimal length for achieving a minimum half-wavelength voltage at high frequency.

From the analysis of both RF and optical characteristics in the previous section, the electro-optic modulation can be characterized based on the DC half-wavelength voltage- length product and the electro-optic response. The DC halfwavelength voltage-length product is strongly dependent on the mode overlap integral factor, $\Gamma$, that is inherently related to the RF and optical mode concentration and overlap, as well as the EO coefficients. To evaluate these expressions, the $\mathrm{EO}$ coefficient of the EO polymer is assumed to be $r_{33}=$ 30,100 , or $170 \mathrm{pm} / \mathrm{V}[8,11,22,28]$. The resulting $V_{\pi} L$ is listed accordingly in Table 1, indicating nearly two orders of magnitude enhancement in EO modulation compared to traditional $\mathrm{LiNbO}_{3}$ traveling wave modulators. Figure 4 depicts the $\mathrm{EO}$ response of the proposed modulators with two different lengths: $L=1$ and $2 \mathrm{~cm}$, obtained from (4). Minimal RF propagation loss and good index matching provide an ultrabroadband electro-optic response, that is, $250 \mathrm{GHz}$. The frequency-dependent driving voltage, derived from the DC $V_{\pi} L$ and electro-optic response $m\left(f_{m}\right)$, demonstrates a subvolt driving voltage, at ultra-high frequencies. The push-pull configuration of the modulator will further reduce the DC $V_{\pi}$ by a factor of 2 if an intensity modulation is interested.

Compared to conventional traveling wave modulators, the proposed dual vertical slot waveguide EO modulators demonstrate superior advantages from a variety of aspects. The extremely strong RF and optical modes confinement within a nanoslot lead to a tight mode overlap, enhancing nonlinear interaction of EO polymer. The reduced RF loss due to a large electrode gap improves the electrooptic response, thereby enabling high speed operation. The application of exotic organic EO polymer owing to its high EO coefficient further lower the driving voltage, therefore enhancing sensitivity. In addition to these, the application of EO property of polymer requires careful material preparation, including EO activation. In order to activate the EO property, $\mathrm{EO}$ polymer is required to pole at an appropriate temperature with an extremely high field, that is, $50 \mathrm{~V} / \mu \mathrm{m}[22]$. The proposed design may offer potential in the EO material poling process. An applied DC bias on the electrodes can be directly extended across the slot. For a given $120-\mathrm{nm}$ slot and $40-\mathrm{V}$ applied voltage, an electric field about $44 \mathrm{~V} / \mu \mathrm{m}$ can be produced. The small slot size and the strong field established with a low applied voltage make an in situ poling process of the polymer feasible.

\section{Conclusion}

In summary, we have presented an integrated RF and optical vertical dual slot waveguide traveling wave EO modulator. By harnessing the unique material properties of the ferroelectric $\mathrm{LiNbO}_{3}$ material and organic EO polymers, the proposed design is able to tightly concentrate both RF and optical modes in a slot for maximizing the nonlinear interaction with the applied electric field. Numerical simulations are performed to characterize the design. The tight mode overlap and large EO coefficient of polymer significantly leverage the modulation sensitivity, leading to a subvolt driving voltage over a length of $1 \mathrm{~cm}$. The improved electrode design significantly reduce the RF propagation attenuation, thereby enabling the design operate over the frequencies of $250 \mathrm{GHz}$. 


\section{Acknowledgments}

The authors gratefully acknowledge Drs. Attila Szep, Robert Nelson, and Gernot Pomrenke from Air Force Research Lab for the valuable discussions and financial support for this research.

\section{References}

[1] J. Macario, P. Yao, R. Shireen, C. A. Schuetz, S. Y. Shi, and D. W. Prather, "Development of electro-optic phase modulator for $94 \mathrm{GHz}$ imaging system," Journal of Lightwave Technology, vol. 27, pp. 5698-5703, 2009.

[2] K. Noguchi, O. Mitomi, and H. Miyazawa, "Millimeterwave Ti: $\mathrm{LiNbO}_{3}$ optical modulators," Journal of Lightwave Technology, vol. 16, no. 4, pp. 615-619, 1998.

[3] Y. Liao, H. Zhou, and Z. Meng, "Modulation efficiency of a $\mathrm{LiNbO}_{3}$ waveguide electro-optic intensity modulator operating at high microwave frequency," Optics Letters, vol. 34, no. 12, pp. 1822-1824, 2009.

[4] Y.-Q. Lu, M. Xiao, and G. J. Salamo, "Wide-bandwidth high-frequency electro-optic modulator based on periodically poled $\mathrm{LiNbO}_{3}$," Applied Physics Letters, vol. 78, no. 8, pp. 10351037, 2001.

[5] D. Janner, M. Belmonte, and V. Pruneri, "Tailoring the electrooptic response and improving the performance of integrated $\mathrm{LiNbO}_{3}$ modulators by domain engineering," Journal of Lightwave Technology, vol. 25, no. 9, pp. 2402-2409, 2007.

[6] Q. Y. Lu, W. H. Guo, D. Byrne, and J. F. Donegan, "Design of low V-pi high-speed GaAs travelling-wave electrooptic phase modulators using an n-i-p-n structure," IEEE Photonics Technology Letters, vol. 20, pp. 1805-1807, 2008.

[7] M. Jarrahi, T. H. Lee, and D. A.B. Miller, "Wideband, low driving voltage traveling-wave Mach-Zehnder modulator for RF photonics," IEEE Photonics Technology Letters, vol. 20, no. 7, pp. 517-519, 2008.

[8] Y. Enami, C. T. Derose, D. Mathine et al., "Hybrid polymersolgel waveguide modulators with exceptionally large electrooptic coefficients," Nature Photonics, vol. 1, no. 3, pp. 180-185, 2007.

[9] Y. Enami, D. Mathine, C. T. Derose et al., "Hybrid crosslinkable polymer/sol-gel waveguide modulators with $0.65 \mathrm{v}$ half wave voltage at $1550 \mathrm{~nm}, "$ Applied Physics Letters, vol. 91, no. 9, Article ID 093505, 3 pages, 2007.

[10] M. Lee, H. E. Katz, C. Erben et al., "Broadband modulation of light by using an electro-optic polymer," Science, vol. 298, no. 5597, pp. 1401-1403, 2002.

[11] C. T. DeRose, D. Mathine, Y. Enami et al., "Electrooptic polymer modulator with single-mode to multimode waveguide transitions," IEEE Photonics Technology Letters, vol. 20, no. 12, pp. 1051-1053, 2008.

[12] E. M. McKenna, A. S. Lin, A. R. Mickelson, R. Dinu, and D. Jin, "Comparison of $\mathrm{r}_{33}$ values for AJ404 films prepared with parallel plate and corona poling," Journal of the Optical Society of America B, vol. 24, no. 11, pp. 2888-2892, 2007.

[13] T. Gorman, S. Haxha, and J. J. Ju, "Ultra-high-speed deeply etched electrooptic polymer modulator with profiled cross section," Journal of Lightwave Technology, vol. 27, no. 1, pp. 68-76, 2009.

[14] L. R. Dalton, P. A. Sullivan, and D. H. Bale, "Electric field poled organic electro-optic materials: state of the art and future prospects," Chemical Reviews, vol. 110, no. 1, pp. 25-55, 2010.
[15] V. R. Almeida, Q. Xu, C. A. Barrios, and M. Lipson, "Guiding and confining light in void nanostructure," Optics Letters, vol. 29, no. 11, pp. 1209-1211, 2004.

[16] R. Sun, P. Dong, N.-N. Feng et al., "Horizontal single and multiple slot waveguides: optical transmission at $\lambda=1550$ nm," Optics Express, vol. 15, no. 26, pp. 17967-17972, 2007.

[17] C. Koos, P. Vorreau, T. Vallaitis et al., "All-optical high-speed signal processing with silicon-organic hybrid slot waveguides," Nature Photonics, vol. 3, no. 4, pp. 216-219, 2009.

[18] F. Dell'Olio and V. M. N. Passaro, "Optical sensing by optimized silicon slot waveguides," Optics Express, vol. 15, no. 8, pp. 4977-4993, 2007.

[19] J.-M. Brosi, C. Koos, L. C. Andreani, M. Waldow, J. Leuthold, and W. Freude, "High-speed low-voltage electro-optic modulator with a polymer-infiltrated silicon photonic crystal waveguide," Optics Express, vol. 16, no. 6, pp. 4177-4191, 2008.

[20] C. A. Barrios, "Ultrasensitive nanomechanical photonic sensor based on horizontal slot-waveguide resonator," IEEE Photonics Technology Letters, vol. 18, no. 22, pp. 2419-2421, 2006.

[21] G. Wang, T. Baehr-Jones, M. Hochberg, and A. Scherer, "Design and fabrication of segmented, slotted waveguides for electro-optic modulation," Applied Physics Letters, vol. 91, no. 14, 2007.

[22] T. Baehr-Jones, B. Penkov, J. Huang et al., "Nonlinear polymer-clad silicon slot waveguide modulator with a half wave voltage of 0.25 v," Applied Physics Letters, vol. 92, no. 16, Article ID 163303, 2008.

[23] M. Hochberg, T. Baehr-Jones, G. Wang et al., "Towards a millivolt optical modulator with nano-slot waveguides," Optics Express, vol. 15, no. 13, pp. 8401-8410, 2007.

[24] K. K. McLauchlan and S. T. Dunham, "Analysis of a compact modulator incorporating a hybrid silicon/electro- optic polymer waveguide," IEEE Journal on Selected Topics in Quantum Electronics, vol. 12, no. 6, pp. 1455-1460, 2006.

[25] S. Shi and D. W. Prather, "Dual rf-optical slot waveguide for ultrabroadband modulation with a subvolt Vp," Applied Physics Letters, vol. 96, Article ID 201107, 2010.

[26] Z. Zhu and T. G. Brown, "Full-vectorial finite-difference analysis of microstructured optical fibers," Optics Express, vol. 10, no. 17, pp. 853-864, 2002.

[27] R. C. Alferness, "Waveguide electrooptic modulators," IEEE Transactions on Microwave Theory and Techniques, vol. 30, no. 8, pp. 1121-1137, 1982.

[28] S.-K. Kim, Y.-C. Hung, W. Yuan et al., "Metal-slotted polymer optical waveguide device," Applied Physics Letters, vol. 90, no. 24, Article ID 243507, 3 pages, 2007. 

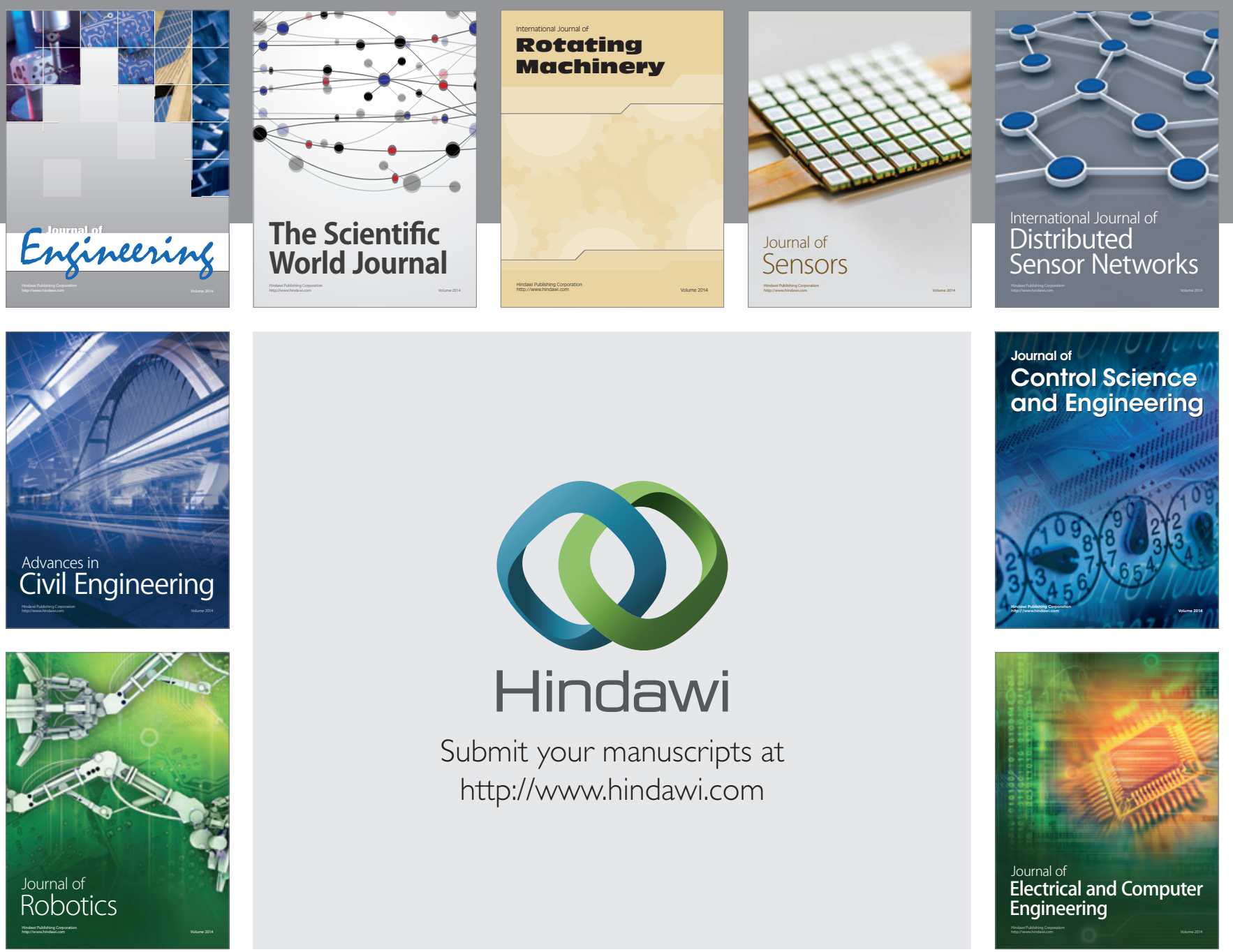

Submit your manuscripts at

http://www.hindawi.com
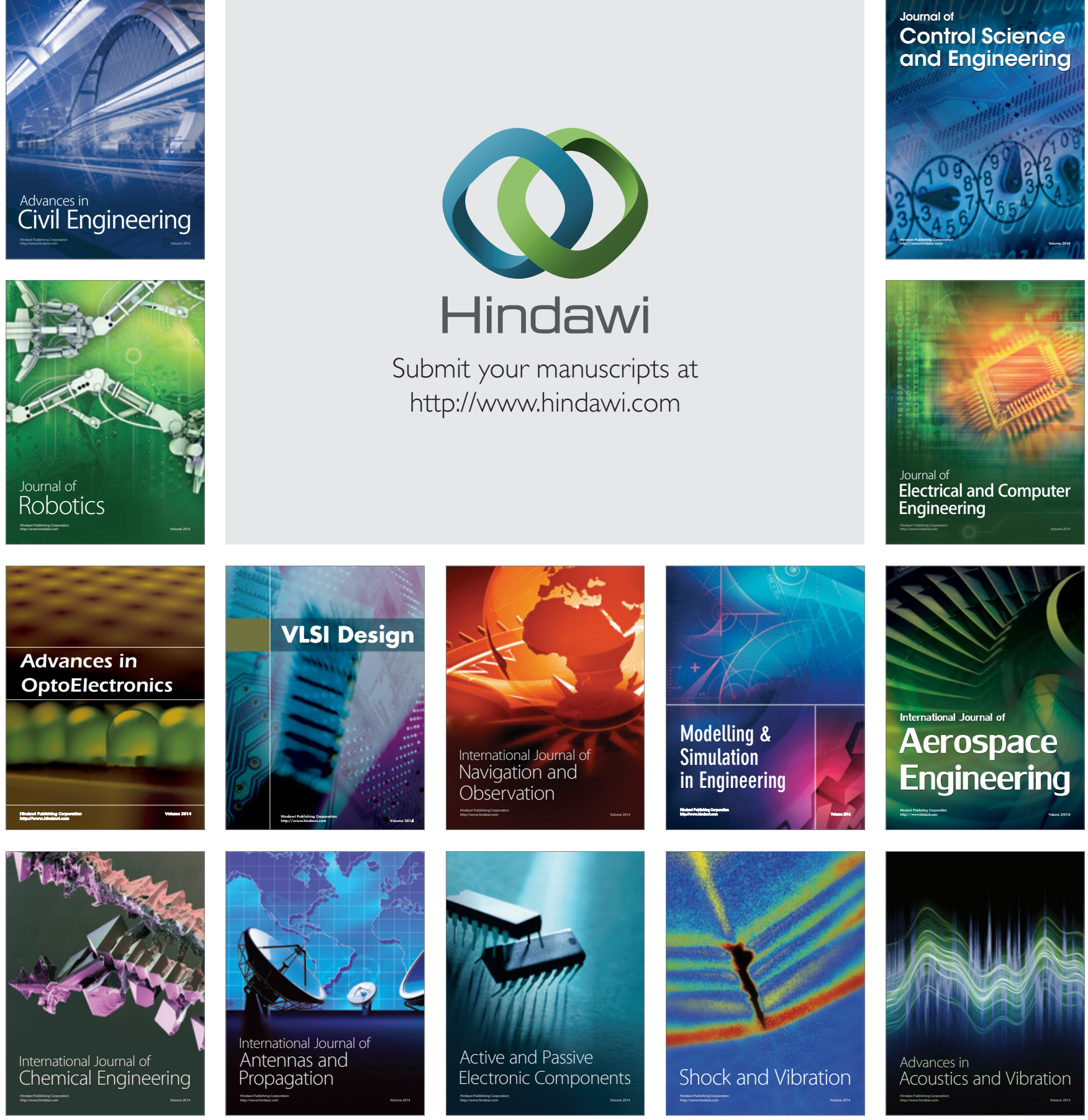